\title{
New Inverse Method for Determining Uniaxial Flow Properties by Spherical Indentation Test
}

\author{
Guoyao Chen, Xiaocheng Zhang, Jiru Zhong ${ }^{*}$, Jin Shi, Qiongqi Wang and Kaishu Guan ${ }^{*}$
}

\begin{abstract}
The spherical indentation test has been successfully applied to inversely derive the tensile properties of small regions in a non-destructive way. Current inverse methods mainly rely on extensive iterative calculations, which yield a considerable computational costs. In this paper, a database method is proposed to determine tensile flow properties from a single indentation force-depth curves to avoid iterative simulations. Firstly, a database that contain numerous indentation force-depth curves is established by inputting varied Ludwic material parameters into the indentation finite elements model. Secondly, for a given experimental indentation curve, a mean square error (MSE) is designated to evaluate the deviation between the experimental curve and each curve in the database. Finally, the true stresses at a series of plastic strain can be acquired by analyzing these deviations. To validate this new method, three different steels, i.e. A508, 2.25Cr1 Mo and 316 $\mathrm{L}$ are selected. Both simulated indentation curves and experimental indentation curves are used as inputs of the database to inversely acquire the flow properties. The result indicates that the proposed approach provides impressive accuracy when simulated indentation curves are used, but is less accurate when experimental curves are used. This new method can derive tensile properties in a much higher efficiency compared with traditional inverse method and are therefore more adaptive to engineering application.
\end{abstract}

Keywords: Spherical indentation test, Database method, Uniaxial stress-strain relationship

\section{Introduction}

Indentation technique is generally take as a non-destructive method with the advantage of local characterization. It provides a feasible approach to determine the mechanical properties for in-service equipment [1-3], welded joints $[2,4-6]$, thin film materials [7-10], etc. The flow properties that describe the plastic hardening behaviors of materials have a widespread application in many cases such as structural design, numerical simulation and thus has been extensively investigated by indentation technique. Traditionally, the flow properties are obtained by establishing a conversion relationship between the indentation response and the elastic-plastic parameters

\footnotetext{
*Correspondence: zhongjiru@outlook.com; guankaishu@ecust.edu.cn School of Mechanical and Power Engineering, East China University of Science and Technology, Shanghai 200237, China
}

of materials with empirical $[1,11,12]$ or analytical approaches [13-17]. However, such procedures are usually complicated due to the geometric and material nonlinearity and a significant contact problem (e.g. the effect of 'pile-up' and 'sink-in'). Moreover, the stress state under the indenter is definitely different from that obtained from uniaxial tensile test, which increases the difficulties for evaluation of the flow curves from the indentation tests.

Recently, with the development of computational algorithms, several reverse strategies incorporating finite element simulations with optimum theory have been proposed. Based on the inverse tools, the material parameters can be directly extracted from the indentation response of materials without any equation that corresponds indentation response to the elastic-plastic parameters of materials. A commonly used tool is 
optimum algorithms [4, 7, 18-23]. Generally, the deviation between experimental and predictive data is considered as the objective function. And then an intelligence algorithm is employed to improve the predictive data by minimizing the objective value using the iterative finite elements (FE) simulations. Luo et al. [20, 21] firstly proposed the use of an optimization approach to extract the elastic-plastic parameters using a single loading-unloading indentation curve. Sun et al. [4] determined the mechanical parameters of the weld zone with the microindentation data where the genetic algorithm (GA) is used for solving the inverse problem. Huang et al. [19] employed the least-square difference between the experimental and simulated force-depth curve as the objective, and identified the materials parameters with a modified particle swarm algorithm (PSO) which increases the ability to obtain a global optimal solution. Note that although these approaches have been widely reported, there are still several limitations. Firstly, an initial guess of material parameters is necessary and usually determined empirically, which have a highly influence on the convergence speed. The improper guess values may impair the accuracy and efficiency of the algorithm. Secondly, it's possible that the iteration end up in a local optimum owing to the drawbacks of algorithms. More significantly, extensive iterative simulations for each parameter identification are required and therefore yield a considerable time and computational cost.

Another efficient inverse tool that are widely used is artificial neural networks (ANN) [24-26]. By adjusting the weights of connections between the artificial neurons, the ANN is trained by the data from finite element analyses. Then, the trained ANN was used to determine the material properties when presented with the indentation force-depth curves. The advantage of an ANN is that the material parameters can be quickly acquired as the corresponding indentation data is inputted. The structure of the ANN (i.e. dimension of the model input, and sizes of the network layer and neurons) has a greatly influence on the predictive ability. However, there is no specific rule for improving the structure of ANNs. What's more, transforming the inverse problem which refers to determine the flow properties from indentation results into a network problem is always difficult.

In this study, a new method is presented to predict the flow properties from a single indention $F$ - $h$ curve where a database is used for solving the inverse problem. The database can replace the iterative algorithms to process the experimental indentation curves, and thus iterative FE simulations are not required in the new method. Firstly, by numerous finite element simulations with constitutive parameters varied systematically in a large range, a database that contain indentation force-depth curves is established. Then, by placing the experimental indentation response into the prepared database, a series of true stress plastic strain points are extracted. The feasibility of this new method was verified through a comparison of predicted tensile properties with the experimental tensile results using three steels.

\section{Materials and Experiments}

Three steels, A508, 2.25Cr1Mo, 316L, which are commonly used in chemical industry, are researched in this study. The optical microstructures are shown in Figure 1, indicating that the microstructure of SA508 steel consists of tempered bainite and 2.25Cr1Mo is bainite with a small amount of ferrite. $316 \mathrm{~L}$ stainless steel is austenite equiaxed grains with a small amount of delta ferrite distributed on the grain boundaries. These steels are manufactured by forging process and therefore regarded as homogeneous and isotropic materials.

The tensile tests were conducted on these steels according to Chinese standard GB/T 228.1-2010. Specimens are fabricated into round bar with gauge radius $8 \mathrm{~mm}$ and length $40 \mathrm{~mm}$. The loading procedures are controlled by displacement with a rate of $0.015 \mathrm{~mm} /$ min at room temperature. Under this loading speed, the strain rate is less than $10^{-5} / \mathrm{s}$, and therefore the tensile tests can be regarded as quasi-static process without the strain-rate effect.

The spherical indentation tests are conducted according to ISO 14577-1 2015 using self-developed machine with a force resolution of $0.5 \mathrm{~N}$ and displacement resolution of $0.1 \mu \mathrm{m}$. The specimens are machined into $50 \mathrm{~mm} \times 10 \mathrm{~mm} \times 10 \mathrm{~mm}$ blocks with the measuring surface paralleled to the bottle surface, and polished by a 2000 grit sandpapers. A tungsten carbide spherical indenter with the diameter of $0.79 \mathrm{~mm}$ is adopted.

ISO 14577-1 2015 indicates that the loading speed of the indenter should be low so that the mechanical properties of the surface are not changed by the impact. For macro range indentations (the penetrate force is greater than $2 \mathrm{~N}$ and less than $30 \mathrm{kN}$ ), it should not exceed $2 \mu \mathrm{m} / \mathrm{s}$. In this study, a vertical displacement rate of $0.03 \mathrm{~mm} / \mathrm{min}$, i.e. $0.5 \mu \mathrm{m} / \mathrm{s}$ is adopted and the maximum penetrate depth is $12 \%$ of the indenter diameter.

Noting that the strain field between the uniaxial tensile tests and indentation tests are totally different, it is necessary that the investigated materials are isotropic and do not have Bauschinger effect. In addition, the true stress-plastic strain curves are structure-independent for the indentation and uniaxial tensile test. 

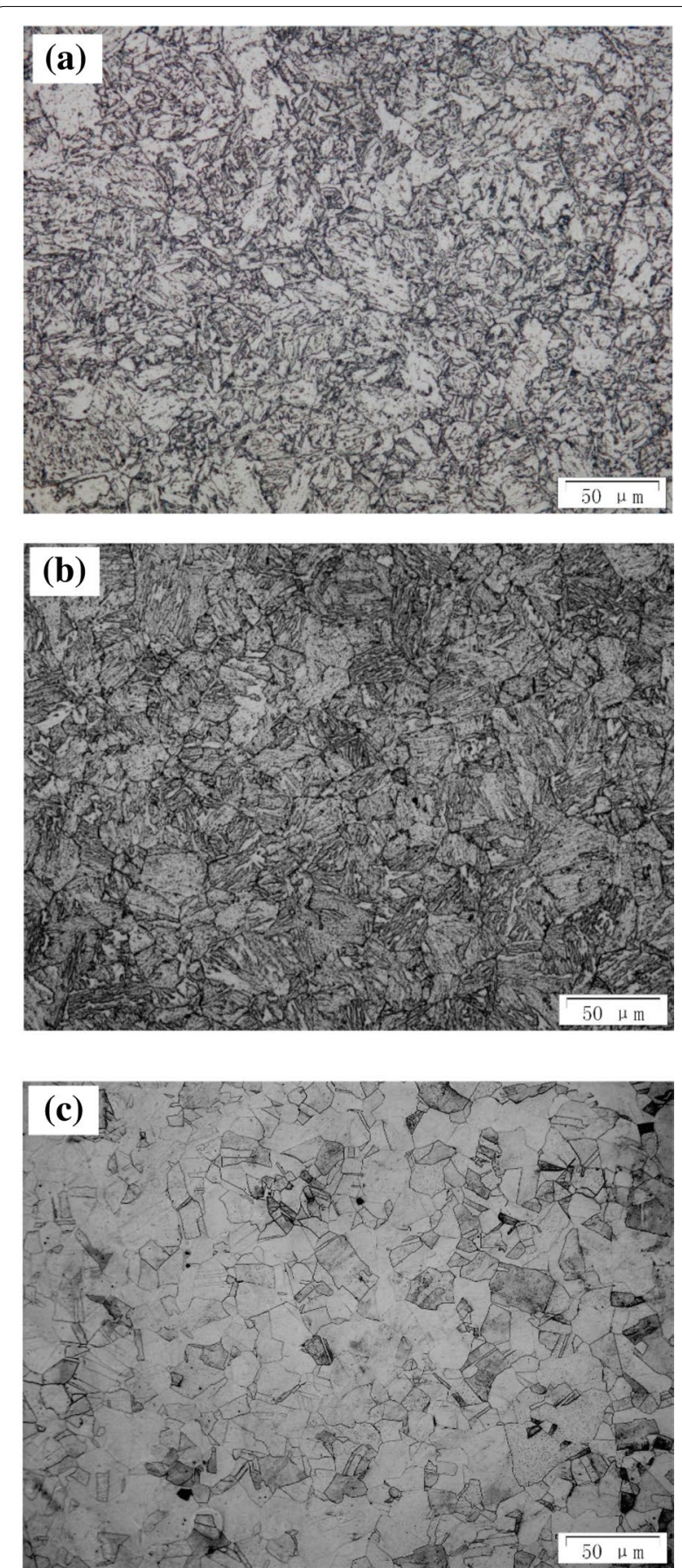

Figure 1 The optical microstructures for (a) A508, (b) 2.25Cr1 Mo and (c) $316 \mathrm{~L}$

\section{Database Approach Modeling}

\subsection{Finite Elements Model}

Taking the features of spherical indentation test into consideration, an axisymmetric model is adopted using ABAQUS/Standard 2018 to reduce the computational time (shown in Figure 2). The spherical indenter with a

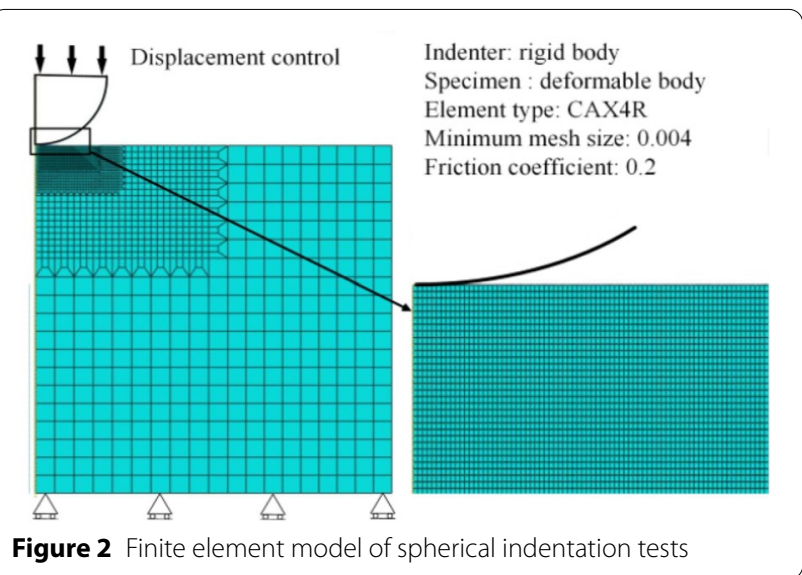

diameter of $0.79 \mathrm{~mm}$ is designated as an analytical rigid body since the deformation is generally ignored compared with the deformation of specimen. The specimen is modelled as a cylinder with the height of $3.5 \mathrm{~mm}$ and the radius of $3 \mathrm{~mm}$. Constraint along the $Y$-axial is imposed to the bottom of the specimen. A vertical displacement was applied to the indenter until the indentation depth reaches to $0.0948 \mathrm{~mm}$, which is consistent with the experiments. The influence of meshing size on the simulated indentation results should be considered. In this study, the meshes beneath the indenter are locally refined. To get the most appropriate mesh size, we discussed three different mesh sizes, $0.002 \mathrm{~mm}, 0.004 \mathrm{~mm}$, and $0.008 \mathrm{~mm}$, and find the model with mesh size of $0.004 \mathrm{~mm}$ is enough to provide the necessary accuracy at reasonable calculation costs. The friction coefficient between the specimen surface and the indenter is fixed at 0.2 according to previous studies [27].

For many steel used in chemical equipment, the Young's module $E$ and Poisson's ratio $v$ are similar. Moreover, previous studies $[28,29]$ have concluded that the indentation curves mainly depend on the plastic flow curves rather than the elastic parameters. Therefore, it is acceptable to take $E$ as $210 \mathrm{GPa}$ and $v$ as 0.3 , respectively $[1,4]$. This allows the focus to be on the behavior of the plastic. A Ludwick hardening model is used to describe the materials hardening behavior:

$$
\sigma=\sigma_{0}+K \varepsilon_{p}^{n},
$$

where $\sigma$ and $\varepsilon_{p}$ are the true stress and true plastic strain, respectively. $\sigma_{0}$ is the initial yield strength, $K$ is the strength coefficient, and $n$ denote the strain hardening exponent.

Thus material constants in the FE model are characterized with a parameter vector $\boldsymbol{x}=\left[\sigma_{0}, K, n\right]^{\mathrm{T}}$. To establish a database that cover a wide range of materials, systematically varied parameter vectors are used to create the 
Table 1 Possible range of parameter vector $\boldsymbol{x}_{j}$ for the database

\begin{tabular}{lccc}
\hline Constitutive model & \multicolumn{2}{l}{ Ludwic } & \\
\cline { 2 - 4 } Parameters & $\boldsymbol{\sigma}_{\mathbf{0}}(\mathrm{MPa})$ & $\boldsymbol{K}(\mathbf{M P a})$ & $\boldsymbol{n}$ \\
\hline Minimum value & 100 & 100 & 0.06 \\
Maximum value & 880 & 1500 & 0.96 \\
Interval & 30 & 30 & 0.03 \\
\hline
\end{tabular}

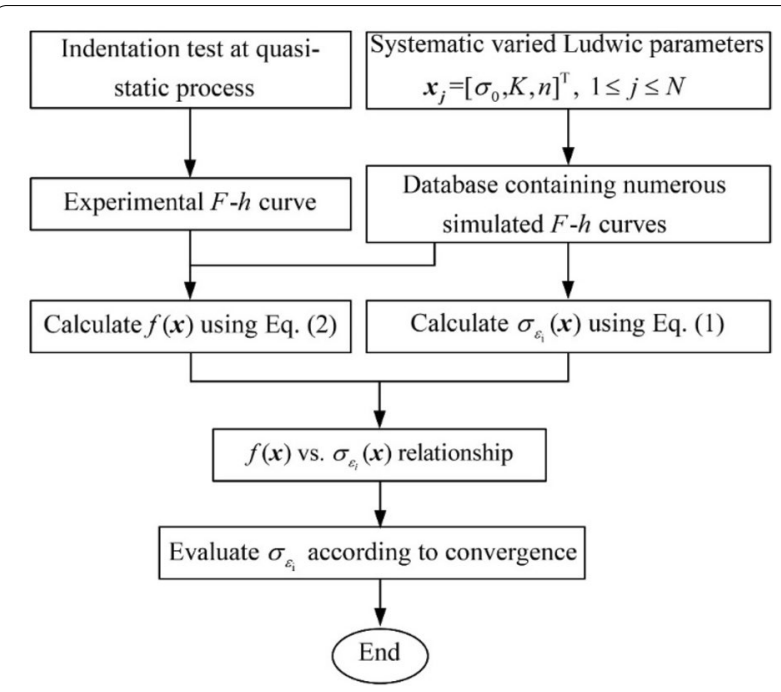

Figure 3 Flow chart for the new inverse method to determine the flow properties of metal materials

Python script files with MATLAB code, and then the Python scripts are run by ABAQUS/Standard to acquire the indentation force-depth curves. Over 20000 parameter vectors denoted by $\boldsymbol{x}_{j}(1 \leq j \leq N)$ are used in this paper. $N$ is the number of vectors in the database. Table 1 shows the range of varied parameters set.

\subsection{Procedures for Uniaxial Flow Properties Determination} Figure 3 shows the schematic illustration for determining the flow properties of metal materials with a single $F-h$ curve based on the application of the database. The FE model in Sect. 3.1 provides the indentation $F$ - $h$ curves of the database. When an experimental forcedepth curve is prepared, a mean square error is used to calculate the difference error between the force-depth curve in the database and that from experiments, which is defined as:

$$
\left\{\begin{array}{l}
f\left(\boldsymbol{x}_{\boldsymbol{j}}\right)=\frac{1}{M} \sum_{i=1}^{M}\left(\frac{F_{i}^{\mathrm{exp}}-F_{i}^{\mathrm{sim}}\left(\boldsymbol{x}_{\boldsymbol{j}}\right)}{F_{i}^{\exp }}\right)^{2} \times 100 \% \\
\boldsymbol{x}_{\boldsymbol{j}}=\left[\sigma_{0}, K, n\right]^{\mathrm{T}} \text { and } 1 \leq j \leq N
\end{array}\right.
$$

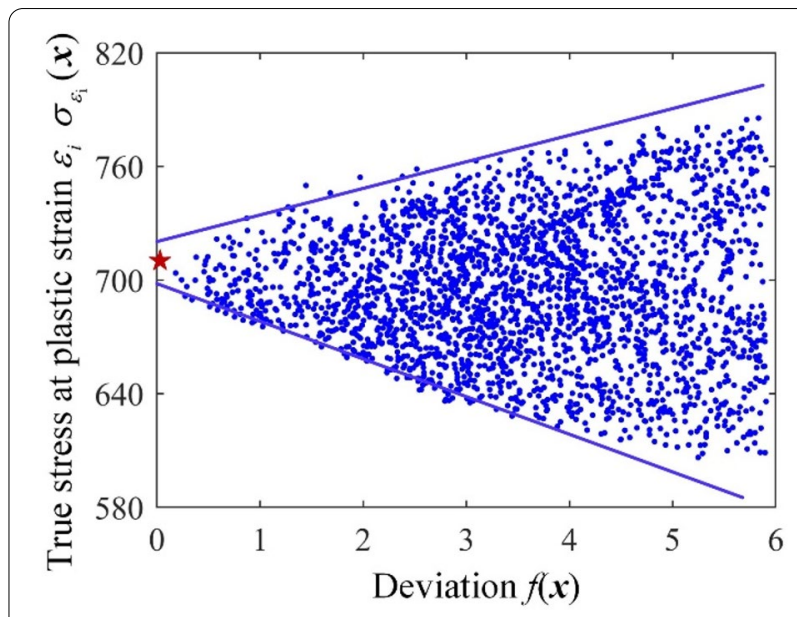

Figure 4 Typical $f(\boldsymbol{x})-\sigma_{\varepsilon_{i}}(\boldsymbol{x})$ relationship indicating convergent $\sigma_{\varepsilon_{i}}(\boldsymbol{x})$ with decreasing $f(\boldsymbol{x})$

where $x_{j}$ represents the $j_{\text {th }}$ materials in the database, $F_{i}^{\exp }$ and $F_{i}^{\text {sim }}\left(\boldsymbol{x}_{\boldsymbol{j}}\right)$ are the indentation forces at the depth of data point $i$ for the experimental material and the $j$ th materials in the database. $M$ is the total number of data points used in the indentation curve.

To determine the flow properties of investigated materials, a series of plastic strain values, namely $\varepsilon_{i}=0.002$, $0.01,0.02,0.04,0.06,0.08,0.1,0.15,0.2,0.3,0.4,0.5$ are selected. The true stress correlated to $\varepsilon_{i}$ for vector $\boldsymbol{x}$ in the database (denoted as $\sigma_{\varepsilon_{i}}(\boldsymbol{x})$ ) can be calculated with Eq. (1). To determine the true stress $\sigma_{\varepsilon_{i}}$ of investigated material, the relation of $f(\boldsymbol{x})$ versus $\sigma_{\varepsilon_{i}}(\boldsymbol{x})$ are investigated in a two-dimensional coordinate system where the $x$ and $y$-axis represent $f(\boldsymbol{x})$ and $\sigma_{\varepsilon_{i}}(\boldsymbol{x})$, respectively. Figure 4 shows the typical $f(\boldsymbol{x})-\sigma_{\varepsilon_{i}}(\boldsymbol{x})$ relationship, which reveals that there exists an approximate linear lower and upper boundaries and the range of $\sigma_{\varepsilon_{i}}(\boldsymbol{x})$ is gradually narrow with decreasing $f(\boldsymbol{x})$. An appropriate explanation for this is that a smaller $f(\boldsymbol{x})$ means a better matched indentation curve with the experiment one and results in a closer $\sigma_{\varepsilon_{i}}(\boldsymbol{x})$ to the value of investigated material. Ideally, when $f(\boldsymbol{x})$ is approximate to zero, $\sigma_{\varepsilon_{i}}(\boldsymbol{x})$ are tended to converge to an exact value, which can be regarded as the true stress correlated to $\varepsilon_{i}$ for the experimental material. Notice that with the facts that the scale of the database is finite and the experimental curves are not definitely noise-free, $f(\boldsymbol{x})$ cannot be zero. Here we specified the lower and upper boundary lines and employ the intersection point of two lines as the true stress of the experimental material. By this means, the stresses at different plastic strains are extracted according to the $f(\boldsymbol{x})$ vs. $\sigma_{\varepsilon_{i}}(\boldsymbol{x})$ relationships. 
As can be seen from the procedures, the database replaced the costly iterative numerical inverse analysis, which therefore provides an easy, and quick route for measuring the flow properties compared with other inverse method. With the database, it only takes dozens of seconds to find the matched true stress-strain curves from the indentation curves. To find the matched materials, the quality of indentation results in the database are crucial. In addition, the database should be large enough to cover the studied materials.

\section{Results and Discussion}

\subsection{Uniaxial Tensile and Indentation Results}

Since the volume is constant in plastic deformation, the true stress-strain curves are derived from the experimental engineering curves:

$$
\left\{\begin{array}{l}
\sigma=\sigma_{\mathrm{e}}\left(1+\varepsilon_{\mathrm{e}}\right) \\
\varepsilon=\ln \left(1+\varepsilon_{\mathrm{e}}\right)
\end{array}\right.
$$

where $\sigma$ and $\varepsilon$ are true stress and strain, $\sigma_{\mathrm{e}}$ and $\varepsilon_{\mathrm{e}}$ are engineering stress and strain.

Figure 5 shows the true stress-strain curves for the three materials, which have definitely different plastic behaviors. Since the segment after necking is not uniform, only the segment before necking are employed. A508 and 2.25Cr1Mo have relatively high yield strengths and low plastic deformation ability. On the contrast, S316L is good ductile material with a lower yield strength. In addition, A508 and 2.25Cr1Mo shows a good power law hardening behaviors, while linear hardening provides a better description for 316L. Figure 5 indicates that the fitting results of the three materials matched their true stress-strain well. The Ludwic fitting parameters are listed in Table 2.

The experimental indentation $F-h$ curves for the three materials are depicted in Figure 6. It is seen that 2.25Cr1Mo has a higher curves than A508 and 316L. This is because $2.25 \mathrm{Cr} 1 \mathrm{Mo}$ has a higher yield stress. The corresponding simulated $F$ - $h$ curves where Ludwic parameters listed in Table 2 are employed as inputs of FE model are also presented (the solid lines). The results show that the simulated $F$ - $h$ curves, in general, agreed well with the corresponding experimental curves. This indicates that the FE model in this paper is accurate enough to generate the indentation curves in the database. For $316 \mathrm{~L}$, the simulated indentation data is a little higher than experimental one. This may result from the fitting error of tensile curves

\subsection{Prediction of Uniaxial Flow Properties}

With the proposed database method, uniaxial flow properties are determined from a single indention $F$ - $h$ curve. Here both indentation force-depth curves extracted from
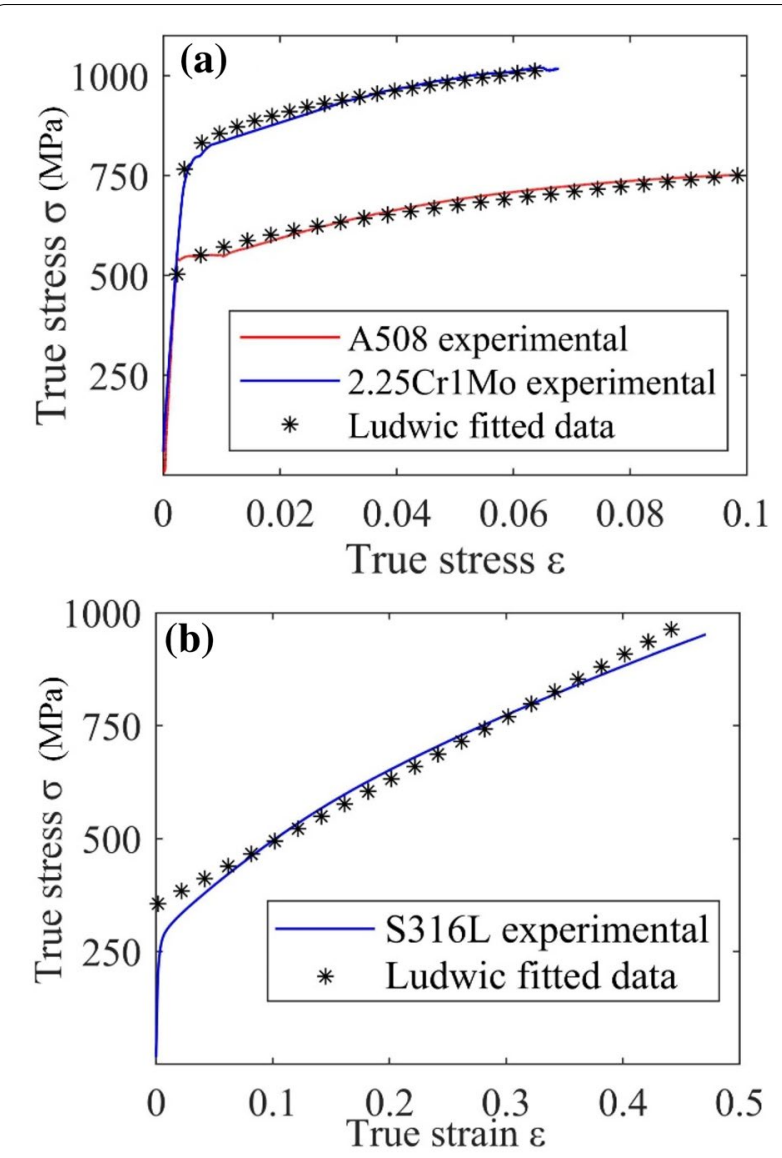

Figure 5 True stress strain curves extracted from uniaxial tensile tests: (a) A508 and 2.25Cr1 Mo; (b) S316L

Table 2 Ludwic parameters of three materials fitted by experimental results

\begin{tabular}{llll}
\hline Ludwic parameters & $\boldsymbol{\sigma}_{0}(\mathrm{MPa})$ & $\boldsymbol{K}(\mathrm{MPa})$ & $\boldsymbol{n}$ \\
\hline A508 & 503.6 & 838 & 0.521 \\
2.25Cr1Mo & 766.3 & 848.1 & 0.440 \\
S316L & 355.8 & 1381 & 1 \\
\hline
\end{tabular}

FE model and measured from experiments are employed as the inputs. Actually, many previous studies applied numerical results to validate the accuracy of a new proposed model. The numerical results do not contain noise in experiments and thus can better evaluate the feasibility of the model.

Figure 7 shows the predicted results when the simulated indentation data of three materials are used as inputs. The asterisks represent the stresses obtained from the relationship of $f(\boldsymbol{x})$ versus $\sigma_{\varepsilon_{i}}(\boldsymbol{x})$. The solid lines represent fitted results of experimental data. Considering 


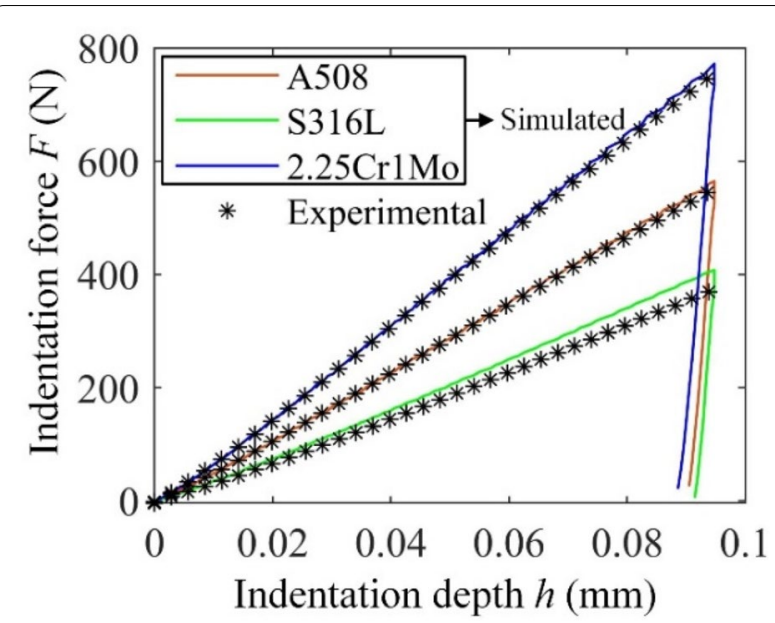

Figure 6 Indentation force-depth curves measured from experiments and extracted from FE model with material parameters presented

that A508 and 2.25Cr1Mo have a lower plastic deformation ability than $316 \mathrm{~L}$, the true plastic strain larger than 0.1 for A508 and 2.25Cr1Mo are not included. To avoid repentance, partial $f(\boldsymbol{x})-\sigma_{\varepsilon_{i}}(\boldsymbol{x})$ relationships are presented in the imagines. It can be seen that the minimum deviation $f(\boldsymbol{x})$ is less than $0.2 \%$, which indicate the $f(\boldsymbol{x})-\sigma_{\varepsilon_{i}}(\boldsymbol{x})$ relationships show good convergence. The predicted stresses of the three materials are in excellent agreement with the fitted tensile data.

Figure 8 shows the predicted stresses when the experimental indentation $F-h$ curves of the three materials are used as inputs. The solid lines represent the experimental flow curves. The minimum $f(x)$ is about $1 \%$. As expected, the convergence is inferior to the result of simulated curves as inputs. As explained in Ref. [[30]], when employing the experimental indentation $F$ - $h$ curves, the quality of the data may be impaired owing to inevitable noise, which results in the intrinsic deviation between the experimental curves and the curves in the database. Nevertheless, the predicted stress values still follow the experimental flow curves reasonably well.

Moreover, it is seen from the result of S316L (Figures 7 (c) and 8(c)) that the predicted stresses at $\varepsilon_{i}=0.4$ and 0.5 have larger errors than others. A possible explanation is that the plastic deformation of region underneath the indenter is constraint by surrounding materials, and thus the overall plastic strain is limited at a small range. Figure 9 depicts the distribution of equivalent plastic strain of a typical indentation simulation. It is seen that the strain larger than 0.5 is distributed in the region underneath the indenter between $0^{\circ}-45^{\circ}$, taking a small region of the whole deformation region. Therefore, the flow curves at larger strain has little contributions to the
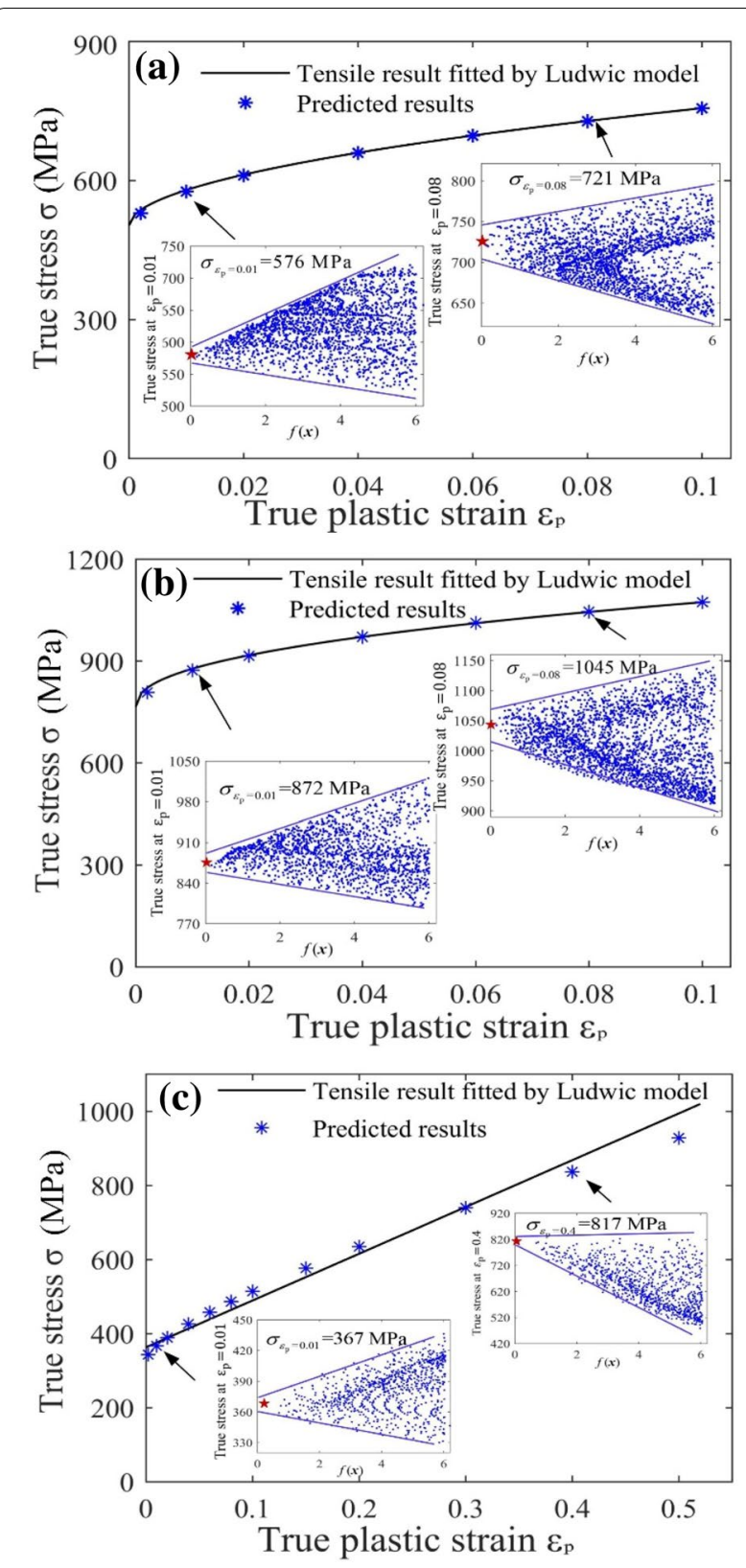

Figure 7 Flow curves fitted from experimental tensile results and predicted using database method with simulated indentation data as inputs for (a) A508, (b) 2.25 Cr1 Mo and (c) 316 L

indentation $F$ - $h$ curves. When indention curves are used to derive the uniaxial flow properties inversely, larger errors may occur at larger plastic strains.

The material parameters fitted by predicted results are given in Table 3. It is seen that the simulated indentation data provide a more accurate material parameters than the experimental indentation data. Moreover, it is interesting to notice that material 

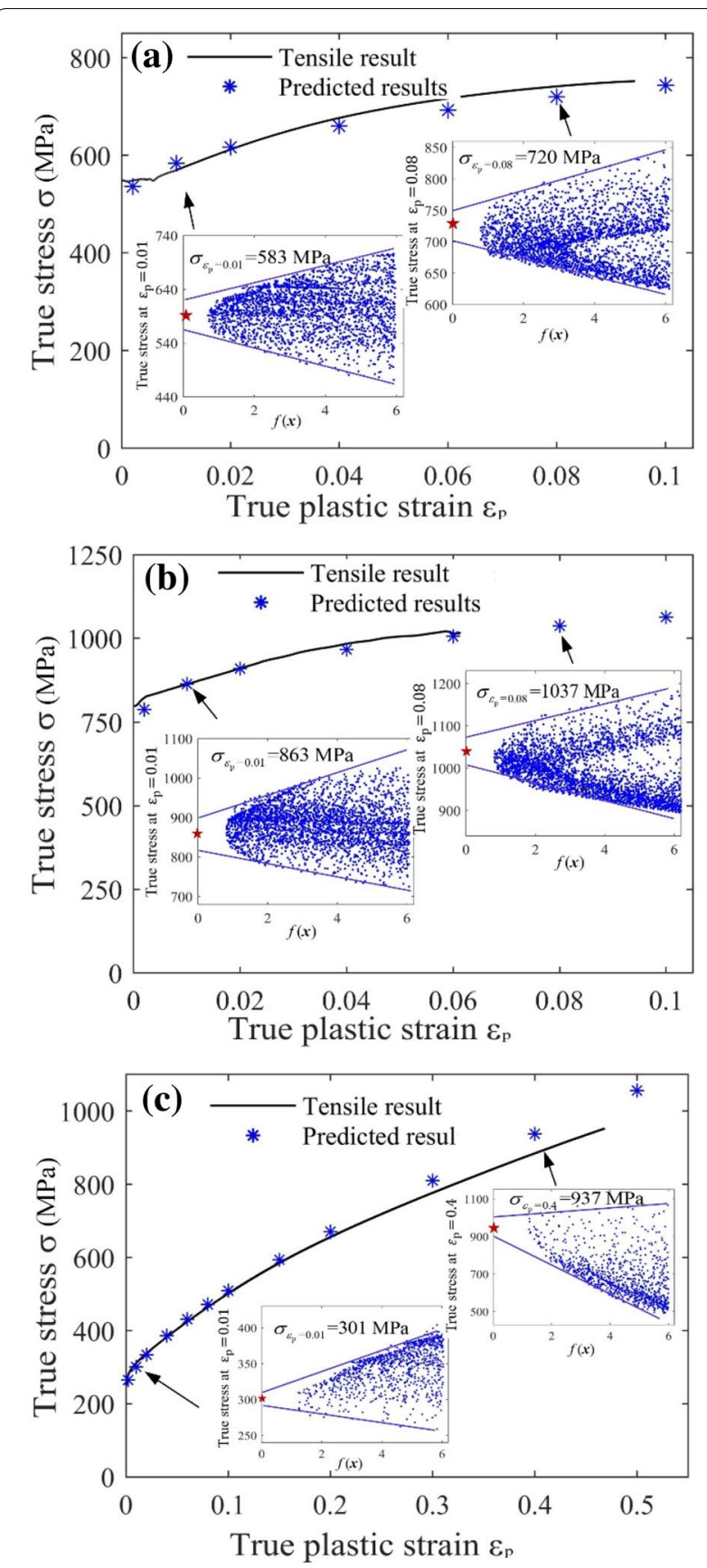

Figure 8 Flow curves measured from experimental tensile results and predicted using database method with experimental indentation data as inputs for (a) A508, (b) 2.25Cr1 Mo and (c) 316 L

parameters determined from simulated and experimental indentation data have considerable differences although the simulated and experimental curves are

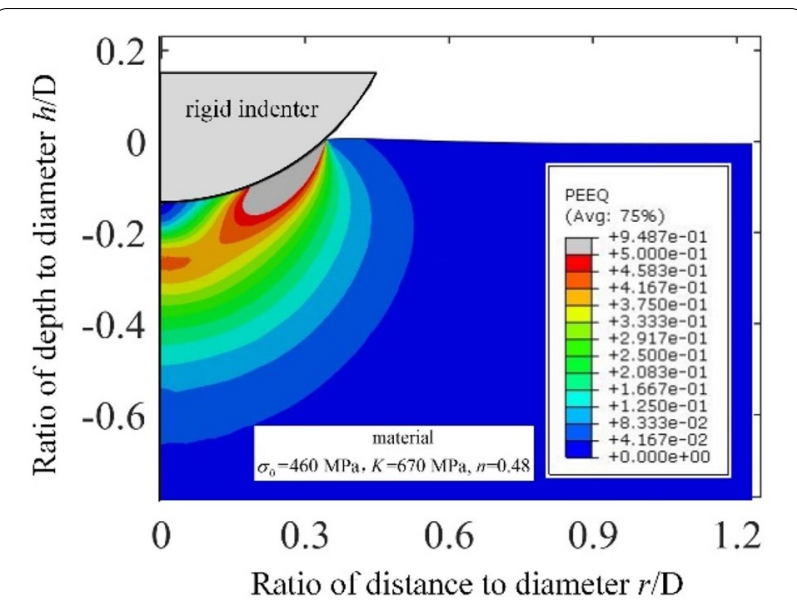

Figure 9 The distribution of equivalent plastic strain

quite consistent (see Figure 6). This reveals that when a single indentation $F-h$ curve are employed to inversely determine the tensile curve, a small difference in $F-h$ curves may account for a large deviation of the tensile curves. For this reason, high-resolution experimental indentation curves that reflect the true elastic-plastic response of materials is necessary when inverse approaches are used.

\section{Conclusions}

In the present study, a new approach was suggested for extraction of material uniaxial flow curves by employing a single spherical indentation test incorporated with the database. Three different steels i.e. A508, 2.25Cr1Mo, S316L are employed to validate the accuracy of the proposed approach. To make a comparison research, both experimental and simulated indentation force-depth curves are used as inputs. The following conclusion can be drawn according to the researches above:

(1) The database method can provide high-quality predictions when simulated indentation $F$ - $h$ curves are used as inputs. When experimental $F-h$ curves are employed, the predicted ability is inferior but follow the tensile flow curves reasonable well.

(2) The tensile curves at larger strain has little contributions to the indentation curves, resulting in the predictive ability of stresses at larger strain are impaired.

(3) A small difference in $F-h$ curves may account for a large deviation of the tensile curves, when inverse 
Table 3 Material parameters fitted from the stress-strain data

\begin{tabular}{|c|c|c|c|c|c|c|c|c|c|}
\hline \multirow[b]{2}{*}{ Material } & \multicolumn{3}{|c|}{ Predicted (input: FE data) } & \multicolumn{3}{|c|}{ Predicted (input: experimental data) } & \multicolumn{3}{|c|}{ Experimental } \\
\hline & $\sigma_{0}$ & $K$ & $n$ & $\sigma_{0}$ & $K$ & $n$ & $\sigma_{0}$ & $K$ & $n$ \\
\hline A508 & 493 & 834 & 0.50 & 495 & 706 & 0.46 & 503 & 838 & 0.52 \\
\hline $2.25 \mathrm{Cr} 1 \mathrm{Mo}$ & 736 & 839 & 0.39 & 674 & 804 & 0.31 & 766 & 848 & 0.44 \\
\hline $316 L$ & 379 & 1155 & 1 & 322 & 1555 & 1 & 355 & 1381 & 1 \\
\hline
\end{tabular}

approaches are used to determine the tensile curve from a single indentation $F-h$ curve.

\section{Acknowledgements}

Not applicable.

\section{Authors' Contributions}

GC and XZ were in charge of the whole trial. GC wrote the manuscript. JZ and KG gave the methodology of this manuscript. JS and QW assisted with sampling and laboratory analyses. All authors read and approved the final manuscript

\section{Authors' Information}

Guoyao Chen, is currently a PhD candidate at School of Mechanical and Power Engineering, East China University of Science and Technology, China. His research field is mechanical properties characterization with indentation tests.

Xiaocheng Zhang, received his PhD degree from East China University of Science and Technology in 2021. He is currently a postdoctoral researcher in Key Laboratory of Pressure Systems and Safety in Shanghai.

Jiru Zhong received his PhD degree from East China University of Science and Technology in 2019. He is currently a postdoctoral researcher in Key Laboratory of Pressure Systems and Safety in Shanghai. His research interests include small specimen test techniques, Safety assessment and life prediction for chemical equipment.

Kaishu Guan received his PhD degree from East China University of Science and Technology, China in 2000. He is now a doctoral supervisor. Professor Guan's research interests include integrity of pressured equipment, mainly covering miniature specimen testing techniques (small punch test and indentation test), and failure analysis and residual life assessment. He participated in the establishment of the standards of small punch tests in China. As a specialist of Shanghai special equipment accident emergency center, he has completed hundreds of failure analysis cases.

Qiongqi Wang is currently a lecturer at East China University of Science and Technology, China. His researches include integrity of pressured equipment and failure analysis.

Jin Shi is currently a PhD candidate at East China University of Science and Technology, China.

\section{Funding}

Supported by China Postdoctoral Science Foundation (Grant No. 2019M661406).

\section{Competing Interests}

The authors declare no competing financial interests.

Received: 31 December 2020 Revised: 23 August 2021 Accepted: 10 September 2021

Published online: 07 October 2021

\section{References}

[1] TS Byun, J H Hong, F M Haggag, et al. Measurement of through-thethickness variations of mechanical properties in SA508 Gr.3 pressure vessel steels using ball indentation test technique, International Journal of Pressure Vessels and Piping, 1997, 74(3): 231-238.

[2] A R H Midawi, C H M Simha, A P Gerlich. Assessment of yield strength mismatch in X80 pipeline steel welds using instrumented indentation. International Journal of Pressure Vessels and Piping, 2018, 168: 258-268.

[3] G. Golański, A Zieliński, M Sroka. Microstructure and mechanical properties of TP347HFG austenitic stainless steel after long-term service. International Journal of Pressure Vessels and Piping, 2020, 188: 104160.

[4] G Sun, F Xu, G Li, et al. Determination of mechanical properties of the weld line by combining micro-indentation with inverse modeling. Computational Materials Science, 2014, 85: 347-362.

[5] R Pamnani, V Karthik, T Jayakumar, et al. Evaluation of mechanical properties across micro alloyed HSLA steel weld joints using automated ball indentation. Materials Science and Engineering: A, 2016, 651: 214-223.

[6] X Chen, Z Lei, Y Chen, et al. Nano-indentation and in-situ investigations of double-sided laser beam welded 2060-T8/2099-T83 Al-Li alloys T-joints. Materials Science and Engineering: A, 2019, 756: 291-301.

[7] N Noii, I Aghayan. Characterization of elastic-plastic coated material properties by indentation techniques using optimisation algorithms and finite element analysis. International Journal of Mechanical Sciences, 2019, 152: 465-480.

[8] C Wang, Q P Cao, T Feng, et al. Indentation size effects of mechanical behavior and shear transformation zone in thin film metallic glasses. Thin Solid Films, 2018, 646: 36-43.

[9] II Argatov, F M Borodich, S A Epshtein, et al. Contact stiffness depthsensing indentation: Understanding of material properties of thin films attached to substrates. Mechanics of Materials, 2017, 114: 172-179.

[10] Y Liu, H Ma, Y Wei, et al. Size effect investigation of indentation response of stiff film/compliant substrate composite structure. International Journal of Solids and Structures, 2020, 193-194: 106-116.

[11] S Wu, TXu, M Song, et al. Mechanical properties characterization of welded joint of austenitic stainless steel using instrumented indentation technique. Materials at High Temperatures, 2016, 33(3): 270-275.

[12] J H Ahn, D Kwon. Derivation of plastic stress-strain relationship from ball indentations: Examination of strain definition and pileup effect. Journal of Materials Research, 2011, 16(11): 3170-3178.

[13] S K Kang, Y C Kim, K H Kim, et al. Extended expanding cavity model for measurement of flow properties using instrumented spherical indentation. International Journal of Plasticity, 2013, 49: 1-15.

[14] H Chen, L X Cai. Theoretical model for predicting uniaxial stress-strain relation by dual conical indentation based on equivalent energy principle. Acta Materialia, 2016, 121: 181-189.

[15] Y Cao. Depth-sensing instrumented indentation with dual sharp indenters: stability analysis and corresponding regularization schemes. Acta Materialia, 2004, 52(5): 1143-1153.

[16] T Zhang, S Wang, W Wang. A constitutive model independent analytical method in determining the tensile properties from incremental spherical indentation tests (ISITs). International Journal of Mechanical Sciences, 2018, 148: 9-19.

[17] K Ito, M Arai. Simple estimation method for strain rate sensitivity based on the difference between the indentation sizes formed by 
spherical-shaped impactors. International Journal of Mechanical Sciences, 2021, 189: 106007.

[18] O Iracheta, C J Bennett, W Sun. A holistic inverse approach based on a multi-objective function optimisation model to recover elastic-plastic properties of materials from the depth-sensing indentation test. Journal of the Mechanics and Physics of Solids, 2019, 128: 1-20.

[19] L Y Huang, K S Guan, T Xu, et al. Investigation of the mechanical properties of steel using instrumented indentation test with simulated annealing particle swarm optimization. Theoretical and Applied Fracture Mechanics, 2019, 102: 116-121.

[20] J Luo, J Lin, T A Dean. A study on the determination of mechanical properties of a power law material by its indentation force-depth curve. Philosophical Magazine, 2006, 86(19): 2881-2905.

[21] J Luo, J Lin. A study on the determination of plastic properties of metals by instrumented indentation using two sharp indenters. International Journal of Solids and Structures, 2007, 44(18-19): 5803-5817.

[22] J J Kang, A A Becker, W Wen, et al. Extracting elastic-plastic properties from experimental loading-unloading indentation curves using different optimization techniques. International Journal of Mechanical Sciences, 2018, 144: 102-109.

[23] A R Hosseinzadeh, A H Mahmoudi. Determination of mechanical properties using sharp macro-indentation method and genetic algorithm. Mechanics of Materials, 2017, 114: 57-68.
[24] A Atrian, G H Majzoobi, S H Nourbakhsh, et al. Evaluation of tensile strength of Al7075-SiC nanocomposite compacted by gas gun using spherical indentation test and neural networks. Advanced Powder Technology, 2016, 27(4): 1821-1827.

[25] K Jeong, H Lee, O M Kwon, et al. Prediction of uniaxial tensile flow using finite element-based indentation and optimized artificial neural networks. Materials \& Design, 2020, 196: 109104.

[26] L Lu, M Dao, P Kumar, et al. Extraction of mechanical properties of materials through deep learning from instrumented indentation. Proceedings of the National Academy of Sciences of the United States of America, 2020, 117(13): 7052-7062.

[27] T Zhang, S Wang, W Wang. An energy-based method for flow property determination from a single-cycle spherical indentation test (SIT). International Journal of Mechanical Sciences, 2020, 171: 105369.

[28] Y P Cao, J Lu. A new method to extract the plastic properties of metal materials from an instrumented spherical indentation loading curve. Acta Materialia, 2004, 52(13): 4023-4032.

[29] M Dao, N Chollacoop, K J Van Vliet, et al. Computational modeling of the forward and reverse problems in instrumented sharp indentation. Acta Materialia, 2001, 49(19): 3899-3918.

[30] G Chen, J Zhong, X Zhang, et al. Estimation of tensile strengths of metals using spherical indentation test and database. International Journal of Pressure Vessels and Piping, 2021, 189: 104284.

\section{Submit your manuscript to a SpringerOpen ${ }^{\circ}$ journal and benefit from:}

- Convenient online submission

$\checkmark$ Rigorous peer review

- Open access: articles freely available online

- High visibility within the field

- Retaining the copyright to your article

Submit your next manuscript at $\boldsymbol{\nabla}$ springeropen.com 